\title{
On the Perturbation of Gibbs Semigroups
}

\author{
N. Angelescu and G. Nenciu \\ Institute for Atomic Physics, Bucharest, Romania \\ M. Bundaru \\ Institute of Physics, Bucharest, Romania
}

Received July 14, 1974

\begin{abstract}
The trace-norm convergence of the Hille-Phillips perturbation series is proved for the whole perturbation class of the generator of a Gibbs semigroup.

In Ref. [1], Uhlenbrock proposed the following terminology:

Definition. A selfadjoint semigroup $\{T(t)\}_{t \geqq 0}$ in a separable Hilbert space with the property:

$$
\operatorname{tr} T(t)<\infty, \quad \forall t>0
$$

is called a Gibbs semigroup; and raised the problem of proving the trace-norm convergence of the Hille-Phillips perturbation series [2] for a conveniently large class of perturbations of the generator of a Gibbs semigroup. He gave also a proof of trace-norm convergence in the case of bounded perturbations, based on an inequality due to Ginibre and Gruber [3].

The aim of this note is to point out that a slight modification of this very argument allows to prove the trace-norm convergence of the series for the whole Hille-Phillips perturbation class.
\end{abstract}

Proposition. Let $T(t)$ be a Gibbs semigroup and $A$ its generator. Let $B$ be A-bounded and such that:

$$
\int_{0}^{1}\|B T(t)\| d t<\infty .
$$

Then the series:

with:

$$
S(t)=\sum_{n=0}^{\infty} S_{n}(t)
$$

$$
S_{0}(t)=T(t) ; \quad S_{n}(t)=\int_{0}^{t} d s S_{0}(t-s) B S_{n-1}(s)
$$

is $\|\cdot\|_{1}$-convergent uniformly for $t$ in compact subsets of $(0, \infty)$. In particular, if $B$ is moreover symmetric, then $S(t)$ is a Gibbs semigroup.

Proof. If $B$ is $A$-bounded, then $B T(t)=[B R(\lambda, A)][(\lambda-A) T(t)]$ is bounded and condition (2) makes sense. One can write $S_{n}(t)$ as a multiple (trace-norm) Bôchner integral:

$$
S_{n}(t)=\int \cdots \int d s_{1} \ldots d s_{n} \chi_{n}^{t}\left(s_{0}, s_{1}, \ldots, s_{n}\right) S_{0}\left(s_{0}\right) B S_{0}\left(s_{1}\right) \ldots B S_{0}\left(s_{n}\right)
$$


where $\chi_{n}^{t}$ is the characteristic function of the set: $s_{i} \geqq 0, i=0, \ldots, n, \sum_{i=0}^{n} s_{i}=t$. Then:

$$
\begin{aligned}
\left\|S_{n}(t)\right\|_{1} \leqq & \int \cdots \int d s_{1} \ldots d s_{n} \chi_{n}^{t}\left(s_{0}, s_{1}, \ldots, s_{n}\right) \\
& \cdot\left\|S_{0}\left(s_{0}\right) B S_{0}\left(s_{1}\right) \ldots B S_{0}\left(s_{n}\right)\right\|_{1} .
\end{aligned}
$$

We shall now use the inequality of Ginibre and Gruber [3]:

$$
\left\|\prod_{i=0}^{n} A_{i} T\left(s_{i}\right)\right\|_{1} \leqq\left(\prod_{i=0}^{n}\left\|A_{i}\right\|\right) \operatorname{tr} T\left(\sum_{i=0}^{n} s_{i}\right)
$$

for every Gibbs semigroup $T(t)$ and bounded operators $A_{0}, \ldots, A_{n}$. We shall take $A_{0}=S_{0}\left(s_{0} / 2\right)$ and $A_{i}=B S_{0}\left(s_{i} / 2\right), i=1,2, \ldots, n$. Denoting $\varphi(t)=\left\|S_{0}(t)\right\|=\exp \left(\omega_{0} t\right)$, and $\psi(t)=\left\|B S_{0}(t)\right\|$, we obtain from (6) and (7):

$$
\left\|S_{n}(t)\right\|_{1} \leqq 2^{n} \cdot \varphi * \psi^{* n}(t / 2) \cdot \operatorname{tr} S_{0}(t / 2)
$$

wherefrom one can proceed as in [2], Theorem 13.4.1, showing that there exist constants $\omega>\omega_{0}$ and $\gamma<1$, such that:

$$
\left\|S_{n}(t)\right\|_{1} \leqq \gamma^{n} \operatorname{tr} S_{0}(t / 2) \exp (\omega t)
$$

which finishes the proof.

Corollary. Let $T(t)$ be a Gibbs semigroup and $A$ its generator. Let $D \subset \mathbb{C}$ be a domain and, for every $z \in D$, let $B(z)$ be given such that:

(i) $B(z)$ is $A$-bounded and $z \sim B(z) T(t)$ is bounded-analytic on $D$ for every $t>0$.

(ii) $\int_{0}^{1} \sup _{z \in D}\|B(z) T(t)\| d t<\infty$.

If $S(z ; t)$ is the semigroup generated by $A+B(z)$, then $z \sim \rightarrow S(z ; t)$ is $\|\cdot\|_{1}$ analytic on $D$ for every $t>0$.

\section{References}

1. Uhlenbrock, D.: J. Math. Phys. 12, 2503 (1971)

2. Hille,E., Phillips, R.S.: Functional analysis and semigroups. Providence, R.I.: Amer. Math. Soc. 1957

3. Ginibre, J., Gruber, C.: Commun. math. Phys. 11, 198 (1969)

Communicated by H. Araki

N. Angelscu

G. Nenciu

Institute for Atomic Physics

Bucharest, Romania

M. Bundaru

Institute of Physics

Bucharest, Romania 